\title{
ChemComm
}

Check for updates

Cite this: Chem. Commun., 2022, 58,3055

Received 15th December 2021 Accepted 26th January 2022

DOI: $10.1039 / \mathrm{d} 1 \mathrm{cc} 07026 f$

rsc.li/chemcomm

\section{Controlled adhesion, membrane pinning and vesicle transport by Janus particles $\dagger$}

\author{
Eleanor J. Ewins, (D) $\ddagger^{*^{a}}$ Koohee Han, (D) $\S^{b}$ Bhuvnesh Bharti, (D) $\boldsymbol{q}^{b}$ Tom Robinson, (D) ${ }^{a}$ \\ Orlin D. Velev $\mathbb{D}^{\mathrm{b}}$ and Rumiana Dimova (D)*a
}

The interactions between biomembranes and particles are key to many applications, but the lack of controllable model systems to study them limits the progress in their research. Here, we describe how Janus polystyrene microparticles, half coated with iron, can be partially engulfed by artificial cells, namely giant vesicles, with the goals to control and investigate their adhesion and degree of encapsulation. The interaction between the Janus particles and these model cell membrane systems is mediated by electrostatic charge, offering a further mode of modulation in addition to the iron patches. The ferromagnetic particle coatings also enable manipulation and transport of the vesicles by magnetic fields.

Interactions of particles with biomembranes are widely studied due to their relevance in multiple current and potential applications, such as in medical imaging, ${ }^{1}$ or as antimicrobial agents, ${ }^{2}$ or to understand the negative environmental impact of microplastics. ${ }^{3}$ In order to take full advantage of these applications, it is important to understand the underlying mechanisms and parameters that govern the adhesion and engulfment of particles by membranes. In cells, this process is referred to as endo- or phagocytosis. Model membrane systems are commonly implemented for studying such processes. ${ }^{4}$ Among these systems are giant unilamellar vesicles (GUVs), ${ }^{5}$ which mimic the cell size and the curvature of the plasma membrane without the compositional complexity of live cells (which includes a wide variety of lipid species, proteins or the associated

\footnotetext{
${ }^{a}$ Max Planck Institute of Colloids and Interfaces, 14476 Potsdam, Germany. E-mail: e.j.ewins@rug.nl,rumiana.dimova@mpikg.mpg.de

${ }^{b}$ Department of Chemical and Biomolecular Engineering, North Carolina State University, Raleigh, NC, 27695, USA

$\dagger$ Electronic supplementary information (ESI) available. See DOI: 10.1039/ d1cc07026f

\# Current address: Department of Biochemistry, Groningen Institute of Biomolecular Sciences \& Biotechnology, University of Groningen, The Netherlands. $\S$ Current address: Department of Chemical Engineering, Kyungpook National University, Daegu, Republic of Korea.

T Current address: Cain Department of Chemical Engineering, Louisiana State University, Baton Rouge, LA 70803, USA.
}

cytoskeleton). ${ }^{6}$ When investigating the parameters that dictate particle-membrane interactions, one vital aspect to consider is the role of the particle properties on the interaction potentials. For example, previous studies have examined how size, shape and surface chemistry impact the interactions of particles with cells $^{7}$ or mimetic systems. ${ }^{4 k, 8}$ What has yet to be explored experimentally is non-receptor mediated interactions between membranes and particles with surface asymmetry.

By using micron-sized Janus particles with two regions of distinctly different surface properties, we investigate to what extent spatially varied surface properties govern the microsphere adhesion and engulfment by GUVs. Such anisotropic particles are of particular interest as they combine two different and sometimes incompatible properties within a single particle. ${ }^{9}$ They also provide means to quantify rotational dynamics due to their broken symmetry, ${ }^{4 j}$ which could be a promising method to study particle endocytosis ${ }^{10}$ or self-propelled guided transport and membrane deformation. ${ }^{4 j, k}$ We find that by spatially altering the particles' surface chemistry, we can control their adhesion and engulfment. We also make use of the iron oxide coating on the particle hemisphere to manipulate particle-vesicle pairs using an external magnetic field gradient.

To select a GUV-particle combination exhibiting adhesion, we first performed high-throughput screening with large unilamellar vesicles (LUVs) of different compositions. The LUVs were prepared via extrusion and incubated with particles of different surface chemistries, see Section S1.2 in the ESI. $\dagger$ LUVs were composed of DOPC with $40 \mathrm{~mol} \%$ either DOPG (negative) or DOTAP (positive) to modulate the membrane charge, see $\mathrm{ESI} \dagger$ for abbreviations and lipid structures. The microparticles used were polystyrene, either functionalised with sulphate or amine groups, resulting in negatively and positively charged surfaces respectively at neutral $\mathrm{pH}$ (here $\mathrm{pH}$ 7.45). The use of fluorescently labelled LUVs, containing 0.5 mol\% Rh-DPPE, allowed for qualitative analysis of the relative affinity between the particle-vesicle combinations.

We observed a clear affinity of the positively charged LUVs to the negative polystyrene particles, see ESI, $\dagger$ Fig. S3. Subsequently, 
we investigated the interactions of positively charged GUVs (containing DOTAP) with uniform and Janus particles exposing a negative (sulphate) surface. The GUVs were prepared via electroformation (ESI, $\dagger$ Section S1.2) from DOPC, doped with 0-5 mol\% DOTAP (above $5 \mathrm{~mol} \%$ the GUV quality and yield was very poor) and $0.5 \mathrm{~mol} \% \mathrm{Rh}-\mathrm{DPPE}$ in $200 \mathrm{mM}$ sucrose. Adhesion to neutral membranes ( $0 \%$ DOTAP) was not observed. The negative charge of the uniform polystyrene particles is from surface sulphate groups.

Iron-patched Janus microspheres were prepared from the uniform particles via metal vapour deposition (see ESI, $\dagger$ Section S1.3) ${ }^{11}$ resulting in a hemispherical patch of $5 \mathrm{~nm}$ of chromium and $20 \mathrm{~nm}$ of iron. Note that the iron patch transforms to iron oxide upon particle resuspension in an aqueous environment. ${ }^{11}$ This patch appears darker in brightfield images, see Fig. S2C (ESI $\dagger$ ).

Both the uniform and Janus particles were dispersed in hypertonic glucose solutions (see ESI, $\dagger$ Section S1 for details); particle incubation with the GUVs generates excess membrane area via osmotic deflation of the vesicles. We observed that this deflation step was necessary for particle engulfment to occur to any extent. Fig. 1 shows example images of the two samples; $5 \%$ DOTAP GUVs that, typically, completely engulf the uniform, negatively charged particles (Fig. 1A), whereas the Janus particles are partially engulfed (half-wrapped) exhibiting pinning of the membrane contact line (Fig. 1B). For the Janus particles, the region of the particle in contact with the membrane is the polystyrene half (light region on particle in Fig. 1B) and the iron-patched half (dark region) remains at the periphery and restricts engulfment. The complete engulfment of the uniform particles suggests strong adhesion of the membrane to the microsphere. This is corroborated by observations showing that the surface of Janus particles is only partially covered by LUVs, see Fig. S4 (ESI $\dagger$ ).

These observations imply that the degree and energy of particle engulfment could be tuneable by altering the

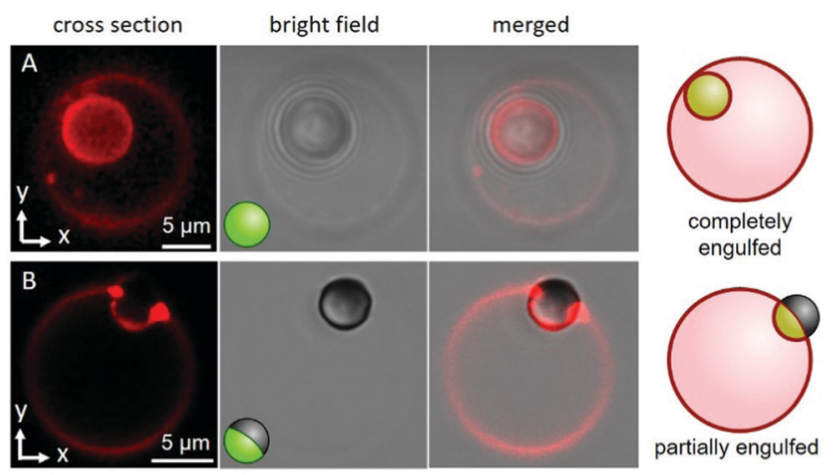

Fig. 1 Confocal cross section and bright-field microscopy images of $5 \%$ DOTAP GUVs (red fluorescence) in contact with microparticles with uniform (A) and Janus (B) surface chemistries. (A) A $6 \mu \mathrm{m}$ negatively charged polystyrene particle is completely engulfed by the GUV. (B) A $4 \mu \mathrm{m}$ Janus particle, half negatively charged polystyrene and half with a thin coating of iron oxide, is partially engulfed by the GUV. The contact line of the adhered vesicle approximately corresponds to the iron oxide-coated region of the particle surface, which can be seen in the brightfield image as the darker region (orientated away from the vesicle surface). The sketches summarise these observations. proportion of the particle surface that has a strong interaction with the membrane, here shown on half-coated (Janus) or uniform particles. We further investigated this concept by measuring how the penetration depth of the particles into vesicles varied, both as a function of the particle surface (uniform or Janus) and membrane charge (either 5 or 1\% DOTAP). These results are displayed in Fig. 2, together with a sketch illustrating the definition of particle penetration depth, $d$, which is comparable to that introduced by Dietrich et al., ${ }^{12}$ who analysed the uptake of uniform particles. Normalisation of $d$ allows us to compare particles and vesicles of different sizes. The images in Fig. 1 show a "close-to-ideal" orientation of the vesicle-particle system, which directly reveals the penetration depth. However, the particles can also exhibit different positions with respect to the vesicle centre, and are typically located at the lower part of the GUV, making it nearly impossible to resolve $d$ from projected images. We thus further develop the approach in ref. 12 taking advantage of the improved resolution of confocal microscopy, especially in the axial direction, see ESI, $\dagger$ Section S3.

The analysis of multiple interactions shows that uniform particles penetrate further into the vesicles than Janus particles do (Fig. 2), as expected from the observations in Fig. 1. The metallic regions supposedly repel the membrane or do not contribute a significant energy gain if the membrane would continue deforming to wet this part of the surface. Therefore, the wetting of the particle surface stops and the particle only partially penetrates into the vesicle. The contact line is pinned

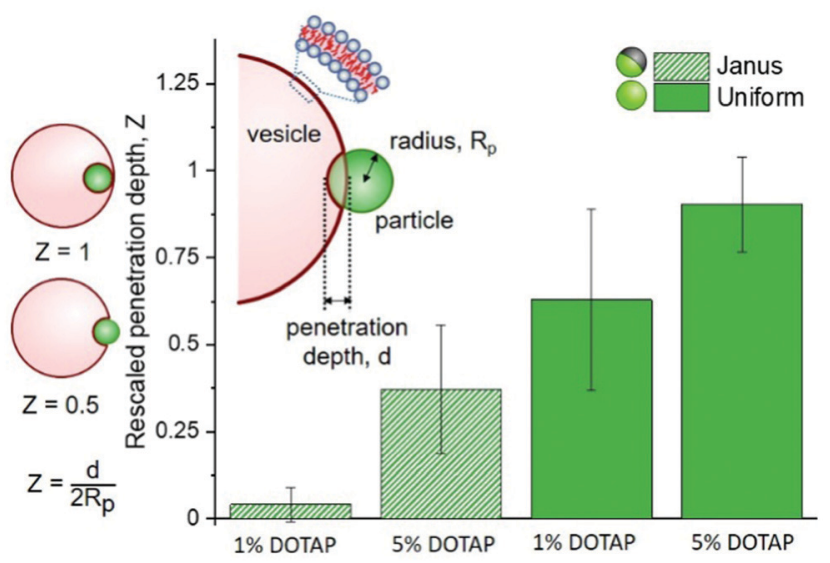

Fig. 2 Penetration depths of Janus and homogeneous particles into GUVs composed of DOPC with 1 or 5\% DOTAP (positively charged). Penetration depth $d$ sketched in the inset is normalised by the particle diameter $2 R_{\mathrm{p}}$, so that different sized particles can be directly compared. The sketches on the left are representative of rescaled penetration depths of $Z=0.5$ and $Z=1$. Janus particles, with half of their surface coated in metal, do not penetrate further than their radial depth into the vesicles $(Z<0.5)$, whereas homogeneous particles penetrate further. Between the two particle types, both particles penetrate further into more positively charged membranes (an effective increase in adhesion energy). Numbers of analyzed vesicle-particle pairs: $1 \%$ DOTAP, Janus $n=8$, uniform $n=7$; $5 \%$ DOTAP, Janus $n=10$, uniform $n=4$. The vesicle diameters were in the range of 10-42 $\mu \mathrm{m}$, and the particle diameters were 3.7-4.05 $\mu \mathrm{m}$ (Janus) and $5.8-6.3 \mu \mathrm{m}$ (uniform). 
at the boundary between the polystyrene and iron oxide. Based on the data shown in Fig. 2, we can also see that there isn't any single definitive penetration depth for each condition. This is most likely due to the challenges that arise from GUV and particle preparation: (i) for GUVs produced from a lipid mixture, it has been shown that the individual vesicle compositions vary, ${ }^{13}$ so does the vesicle size relative to that of the particles. (ii) Vesicles with similar sizes can exhibit variable excess area for wrapping the particles. (iii) The surface chemistry of the polystyrene part of the Janus particles may differ from that of the homogeneous particles because of preparation steps (see ESI, $\dagger$ Section S1.3). (iv) Variation in the membrane spontaneous curvature expected due to charge asymmetry ${ }^{13}$ has been predicted to play a crucial role in particle engulfment. ${ }^{14}$

For both uniform and Janus particles the penetration depths have a dependence on the membrane charge (percentage of positively charged DOTAP, see Fig. 2); essentially, increasing the adhesion energy between the particle and the vesicle results in increased particle penetration. This process is seemingly governed by charge. To further investigate this, we observed the effects of increased ionic strength $(150 \mathrm{mM} \mathrm{NaCl})$ on adhesion of LUVs with DOTAP to uniform particles.

Fig. 3A shows images of particles with adhered LUVs in the presence and absence of salt, see also Fig. S6, ESI. $\dagger$ To assess the effect of salt, we quantified the fluorescence intensity of the particles; see Fig. S7 (ESI $\dagger$ ). In $150 \mathrm{mM} \mathrm{NaCl}$, which is expected to screen the charges, the intensity of the adhered LUVs is roughly 1.8 times lower than that of the samples containing only sugars (0 $\mathrm{mM} \mathrm{NaCl})$, Fig. 3C; the scatter in fluorescence intensity values is possibly due to the small size variation between particles, as all particles are measured at the same distance from the glass surface. The results demonstrate that
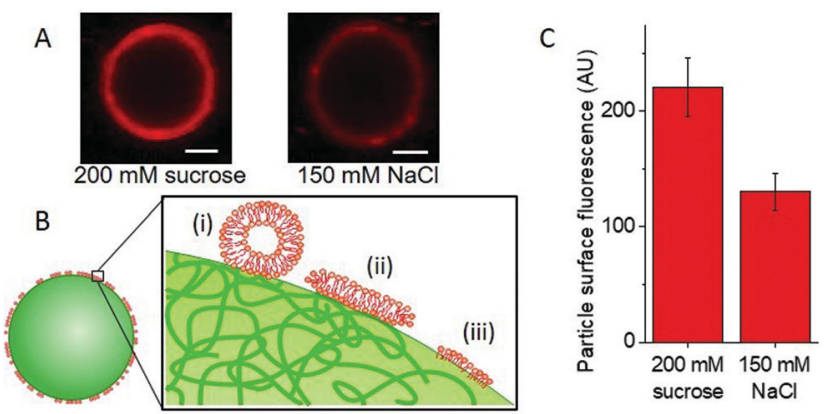

Fig. 3 Effects of salt on the adhesion of positively charged membranes to negatively charged uniform particles. (A) LUVs (100 nm) composed of DOPC/DOTAP/Rh-DPPE 94.5/5/0.5, adhere to negatively charged polystyrene $6 \mu \mathrm{m}$ particles to varying extents depending on the salt concentration in the solution, as deduced from confocal scans (scale bars: $2 \mu \mathrm{m}$ ). (B) The sketch (not to scale) represents possible reorganization of the LUV membrane (red) upon contact with the particle surface (green) leading to non-uniform fluorescence over the particle: (i) LUV adhesion, (ii) vesicle rupture and formation of a supported bilayer, or (iii) restructuring to a monolayer-like structure adhered to more hydrophobic patches on the particle surface. (C) In the presence of sugars, the fluorescence intensity on the particle surface is roughly 1.8 times higher; the presence of salt partially screens the electrostatic LUV-particle interactions. The analysis is performed on 10 particles for each condition. the interactions depend on electrostatics, in correlation with our observations of increased penetration depths in GUVs with a higher DOTAP content.

However, these interactions are not only electrostatic, as we still detect some LUV fluorescence on the particles in the presence of salt. Fig. 3B includes a sketch depicting different possible configurations of the LUV lipids and membrane at the particle surface: (i) docked LUVs (single adhered LUVs appear to produce stronger signal; see Fig. S8, ESI $\dagger$ ); (ii) supported lipid bilayer (shown to form when LUVs adhere to silica particles and collapse ${ }^{15}$ ); and (iii) frustrated lipid monolayer adsorbed onto the hydrophobic regions of the latex surface as speculated by Dietrich et al. ${ }^{12}$

In addition to providing a region with a lower adhesion energy, the iron oxide patch on the Janus particles also attributes magneto-responsiveness. ${ }^{16}$ The particles move towards regions of higher magnetic field intensity (magnetophoresis). This is widely used with uniform magnetic particles in cell sorting protocols. ${ }^{17}$ Indeed, we observe magnetophoresis of the Janus particle-vesicle complex in the presence of a magnetic field (Fig. 4), see ESI for setup details. $\dagger$ GUVs were prepared from DOPC/DOTAP 95/5 mol\%. When the source of the magnetic field is located to the lower left of the chamber, the particle-vesicle pair moves in this direction (Fig. 4A). Conversely, when the magnet is moved to the upper right corner of the chamber, the same particle-vesicle pair changes direction (Fig. 4B). The particle remained adhered to the GUV, and it was possible to repeat similar manipulations with further particle-vesicle pairs, where the distance traversed was

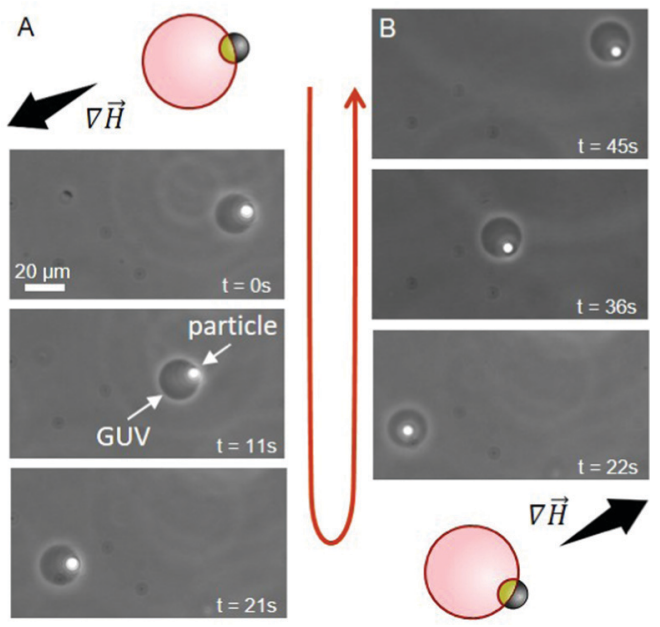

Fig. 4 Time sequence demonstrating the transport of a GUV (dark circle) via manipulation with an adhered Janus particle (white spot) under magnetic field gradient $(\nabla \vec{H})$; phase-contrast microscopy, see also ESI, $\dagger$ Movie S1. The dense particle has sedimented to the lower half of the vesicle (out of focus, appearing inside), but it is outside the GUV. The schematic diagrams indicate the initial particle-vesicle configuration and the magnetic field gradient. (A) The magnet is located to the bottom left, causing the Janus particle to move towards higher field gradient. (B) The magnet is now located to the top right and the Janus particle moves towards this region. The particles are heavy and having both vesicle and particle in focus is not always possible. 
comparable to the size of the observation chamber $(\approx 10 \mathrm{~mm})$. Our approach of transporting vesicles by means of adsorbed Janus particles provides a facile and precise means of moving and sorting GUVs. The approach appears superior in terms of transporting the vesicles over large distances compared to mainly rotating them as shown previously. ${ }^{18}$

In conclusion, we demonstrated the ability to control the extent of particle engulfment by membranes using Janus particles with regions of different affinities for the GUV membrane. We also show that the particle iron oxide coating provides enhanced capabilities for vesicle transport via magnetophoresis.

The degree of penetration depends on both particle surface asymmetry and lipid composition. This is coupled with a decrease in LUV adhesion in the presence of salt, suggesting that this system could be finely tuned to provide the desired degree of particle adhesion and penetration.

The use of Janus particles as a means to separate cell-like objects provides multiple opportunities for further development. The anisotropic surface could be used to limit the cells' exposure to the damaging iron oxide, ${ }^{19}$ by creating regions of higher and lower affinity with the membrane. The exposed, non-binding region of the particle could also be functionalised so as to undergo surface reactions, as another means for generating self-propulsion. ${ }^{20}$ The selective adhesion demonstrated here could also be used as a template for spatially confined lipid sorting, as demonstrated earlier. ${ }^{18}$ The controlled and directional force applied to GUVs could be used for quantitative characterization of the membrane stiffness and moduli. This can be achieved both by the magnetic pull-off and torque. One could also consider a potential use of the entire vesicle-particle ensemble as a drug delivery system, with the manoeuvrability provided by the iron oxide patch, the lipids ensuring biocompatibility and the vesicles serving as a drug transporter.

Open Access funding provided by the Max Planck Society.

\section{Conflicts of interest}

There are no conflicts to declare.

\section{References}

1 X. Mulet, B. J. Boyd and C. J. Drummond, J. Colloid Interface Sci., 2013, 393, 1-20.

2 (a) L. Wang, C. Hu and L. Shao, Int. J. Nanomed., 2017, 12, 1227-1249; (b) G. Ren, D. Hu, E. W. C. Cheng, M. A. Vargas-Reus, P. Reip and R. P. Allaker, Int. J. Antimicrob. Agents, 2009, 33, 587-590.

3 M. Cole, P. Lindeque, C. Halsband and T. S. Galloway, Mar. Pollut. Bull., 2011, 62, 2588-2597.

4 (a) A. S. V. L. C. Peetla, Mol. Pharmaceutics, 2009, 6, 1264-1276; (b) K. L. Chen, Environ. Sci. Technol., 2014, 48, 873-880; (c) H. Kettiger, G. Québatte, B. Perrone and J. Huwyler, Biochim. Biophys. Acta, 2016, 1858, 2163-2170; (d) I. Koltover, J. O. Rädler and C. R. Safinya, Phys. Rev. Lett., 1998, 82, 1991-1994; (e) E. Ewins, R. B. Lira, W. Y. Zhang, J. Y. Yuan, M. Antonietti, T. Robinson and R. Dimova, Adv. Sci., 2019, 6; $(f)$ C. van der Wel, D. Heinrich and D. J. Kraft, Biophys. J., 2017, 113, 1037-1046; $(g)$ S. Li and N. Malmstadt, Soft Matter, 2013, 9, 4969; $(h)$ H. T. Spanke, R. W. Style, C. François-Martin, M. Feofilova, M. Eisentraut, H. Kress, J. Agudo-Canalejo and E. R. Dufresne, Phys. Rev. Lett., 2020, 125, 198102; (i) C. Van Der Wel, N. Bossert, Q. J. Mank, M. G. T. Winter, D. Heinrich and D. J. Kraft, Langmuir, 2017, 33; $(j)$ V. Sharma, E. Azar, A. P. Schroder, C. M. Marques and A. Stocco, Soft Matter, 2021, 17, 4275-4281; (k) H. R. Vutukuri, M. Hoore, C. Abaurrea-Velasco, L. van Buren, A. Dutto, T. Auth, D. A. Fedosov, G. Gompper and J. Vermant, Nature, 2020, 586, 52-56; (l) A. H. Bahrami, R. Lipowsky and T. R. Weikl, Phys. Rev. Lett., 2012, 109, 188102.

5 (a) R. Dimova, S. Aranda, N. Bezlyepkina, V. Nikolov, K. A. Riske and R. Lipowsky, J. Phys.: Condens. Matter, 2006, 18, 1151-1176; (b) P. Walde, K. Cosentino, H. Engel and P. Stano, ChemBioChem, 2010, 11, 848-865; (c) R. Dimova and C. Marques, The giant vesicle book, CRC Press, 2019.

6 (a) Y. H. M. Chan and S. G. Boxer, Curr. Opin. Chem. Biol., 2007, 11, 581-587; (b) S. F. Fenz and K. Sengupta, Integr. Biol., 2012, 4, 982-995.

7 (a) B. D. Chithrani, A. A. Ghazani and W. C. W. Chan, Nano Lett., 2006, 6, 662-668; (b) S. E. A. Gratton, P. A. Ropp, P. D. Pohlhaus, J. C. Luft, V. J. Madden, M. E. Napier and J. M. DeSimone, Proc. Natl. Acad. Sci. U. S. A., 2008, 105, 11613-11618; (c) M. Wu, H. Guo, L. Liu, Y. Liu and L. Xie, Int. J. Nanomed., 2019, 14, 4247-4259; (d) J. Rejman, V. Oberle, I. S. Zuhorn and D. Hoekstra, Biochem. J., 2004, 377, 159-169.

8 (a) S. Zhang, A. Nelson and P. A. Beales, Langmuir, 2012, 28, 12831-12837; (b) M. Bally, A. Gunnarsson, L. Svensson, G. Larson, V. P. Zhdanov and F. Höök, Phys. Rev. Lett., 2011, 107, 188103; (c) K. Shigyou, K. H. Nagai and T. Hamada, Langmuir, 2016, 32, 13771-13777.

9 (a) S. Jiang, Q. Chen, M. Tripathy, E. Luijten, K. S. Schweizer and S. Granick, Adv. Mater., 2010, 22, 1060-1071; (b) A. Walther and A. H. E. Müller, Soft Matter, 2008, 4, 663-668.

10 (a) Y. Gao and Y. Yu, Langmuir, 2015, 31, 2833-2838; (b) Y. Gao and Y. Yu, J. Am. Chem. Soc., 2013, 135, 19091-19094.

11 B. Bharti, D. Rutkowski, K. Han, A. U. Kumar, C. K. Hall and O. D. Velev, J. Am. Chem. Soc., 2016, 138, 14948-14953.

12 C. Dietrich, M. Angelova and B. Pouligny, J. Phys. II, 1997, 7, 1651-1682.

13 J. Steinkühler, P. De Tillieux, R. L. Knorr, R. Lipowsky and R. Dimova, Sci. Rep., 2018, 8, 11838.

14 J. Agudo-Canalejo and R. Lipowsky, ACS Nano, 2015, 9, 3704-3720.

15 R. B. Lira, T. Robinson, R. Dimova and K. A. Riske, Biophys. J., 2019, 116, 79-91.

16 C. Bao, G. Pähler, B. Geil and A. Janshoff, J. Am. Chem. Soc., 2013, 135, 12176-12179.

17 (a) B. D. Plouffe, S. K. Murthy and L. H. Lewis, Rep. Prog. Phys., 2014, 78, 016601; (b) A. Ito, M. Shinkai, H. Honda and T. Kobayashi, J. Biosci. Bioeng., 2005, 100, 1-11.

18 Z. Liu, J. Cui and W. Zhan, Soft Matter, 2020, 16, 2177-2184.

19 (a) M. Kumari, S. Rajak, S. P. Singh, S. I. Kumari, P. U. Kumar, U. S. N. Murty, M. Mahboob, P. Grover and M. F. Rahman, J. Nanosci. Nanotechnol., 2012, 12, 2149-2159; (b) T. R. Pisanic, J. D. Blackwell, V. I. Shubayev, R. R. Fiñones and S. Jin, Biomaterials, 2007, 28, 2572-2581.

20 S. Eloul, W. C. K. Poon, O. Farago and D. Frenkel, Phys. Rev. Lett., 2020, 124, 188001. 